\title{
Modeling of Situation Response Time in Vehicular Ad-Hoc Network
}

\author{
Ajulo, Emmanuel Bamidele, Akinyede, Raphael Olufemi and Adewale, Olumide Sunday
}

\begin{abstract}
In Vehicular Ad-Hoc Networks (VANETs), wireless-equipped vehicles form a network spontaneously while traveling along the road. The direct wireless transmission from vehicle to vehicle makes it possible for them to communicate even where there is no telecommunication infrastructure; this emerging new technology provide ubiquitous connectivity to vehicular nodes while on the move, The main idea is to provide ubiquitous connectivity to vehicular nodes while on the move, and to create efficient vehicle-to-vehicle communications that enable the Intelligent Transportation Systems (ITS). This is achieved by allowing nodes within certain ranges to connect with each other in order to exchange information. Since accident happens in split seconds, to avoid communication inefficiency, there is a need for this information to get to the intended vehicle on time. To solve this problem, this work models each vehicle in a chain of others and how it responds to the traffic around it using Microscopic (also known as carfollowing) method for modeling traffic flow; driver- to-driver and driver-to-road interactions within a traffic stream and the interaction between a driver and another driver on road were considered. The essence of this modeling is to determine the minimum response time required for a vehicle in VANET to respond and communicate situations on the road. A simulated scenario was carried out for two vehicles, a leading vehicle and following vehicle. The result shows that with an average of 32 meters apart with an average difference in velocity of $1.23 \mathrm{~m} / \mathrm{s}$, a minimum of $0.9 \mathrm{secs}$ is required for efficient situation response communication to ensue between them.
\end{abstract}

Index Terms - Vehicular Ad-hoc Network (VANET), Microscopic, Macroscopic, Distance Headway and Distance Gap, Vehicle-to-Vehicle Communication (V2V)

\section{INTRODUCTION}

Road-traffic accidents claim thousands of lives every year in Nigeria, Africa and the world over. Statistics according to the World Health Organization (WHO) shows that over 1.3 million people are estimated to have been killed annually in a road accident while over 50 million people sustain different degrees of injuries from

Manuscript received January 25, 2018. This work was supported in part by Department of Computer Science, The Federal University of Technology, Akure, Nigeria.

Ajulo, Emmanuel Bamidele is with the Department of Computer Science, The Federal University of Technology, Akure, Nigeria; (emailemmanuelajulo@gmail.com)

Akinyede, Raphael Olufemi is with Department of Computer Science, The Federal University of Technology, Akure, Nigeria; (email. roakinyede@futa.edu.ng)

Adewale, Olumide Sunday is with Department of Computer Science, The Federal University of Technology, Akure, Nigeria; (email. Olumide_adewale@futa.edu.ng) such crashes [1]. Further statistics show that over $60 \%$ of all these mishaps take place in suburban and urban areas, and Nigeria is ranked 191 out of 192 countries in the world with unsafe roads and 162 death rates per 100,000 populations from road traffic accident $[2,3,4]$. The probable causes of these mishaps include over speeding vehicles, fewer traffic signals, and road signs, potholes, absence or less numb of speed breakers, and lack of traffic surveillance. In view of this, much significant innovation toward avoiding such deadly traffic mishaps world over has been in the course throughout the years, and concerted efforts have been made to deploy communication capabilities in vehicles and transport infrastructure, leading to Intelligent Transportation Systems (ITS). It is however envisioned that wireless communication will allow both vehicle-tovehicle communication $(\mathrm{V} 2 \mathrm{~V})$ and communication between vehicles and roadsides fixed infrastructures (V2R) to form Vehicular Ad-hoc Networks (VANETs) for exchanging messages carrying both safety and nonsafety information.

In Vehicular Ad-hoc Networks (VANETs), wirelessequipped vehicles form a network spontaneously while traveling along the road. The direct wireless transmission from vehicle to vehicle makes it possible for them to communicate even where there is no telecommunication infrastructure, such as the base stations; vehicles can share up-to-date traffic information on the fly. This is an emerging new technology to integrate the capabilities of new generation wireless networks to vehicles $[5,6]$. The main idea is to provide ubiquitous connectivity to vehicular nodes while on the move, and this is achieved by allowing nodes within certain ranges to connect with each other in order to exchange information. Since accident happens in split seconds, to avoid communication inefficiency; there is a need for this information to get to the intended vehicle on time before the occurrence, thus a need to ascertain the minimum situation response time of vehicles on the network. In the light of this, a mathematical model for situation response time in VANET was developed in this work. The model looks at each vehicle in a chain of others and how it responds to the traffic around it. The essence of this model is to determine the minimum time required for a vehicle to respond to a situation on the road. According to [7] the traffic model is largely divided into two types of models, Macroscopic and Microscopic. Macroscopic models study traffic from an overall or 
average perspective - the flow of vehicles into and out of the system (flux), the average speed of vehicles, or total vehicles, while Microscopic models study the motion of individual vehicles, typically functions of the position, velocity, and acceleration. The behavior of Microscopic models is usually dictated by a lead vehicle, thus, they have termed 'car-following' models and it is the basis for the model used in this work.

\section{REVIEW OF RELATED WORKS}

LWR Model was developed in 1950 and it was named after its creators Lighthill, Whitham, and Richards (LWR). LWR model was based on basic assumptions that velocity depends on, that is, traffic density. The model is followed by AR Model, also named after its creators Aw and Rascle. The AR model attempts to move away from a fluid-flow based model. their argument is that the older macroscopic models have held too closely to the fluid dynamic approach. Then the Zhang Model named after its creator Michael Zhang was introduced. Zhang model moves completely away from fluid behavior. The most basic Microscopic Model called the Gipp's Model was developed in 1970. The model uses driving states to model traffic flow. One disadvantage of Gipp's model was that certain parameters had unrealistic parameters; which may allow unrealistic breaking behavior beyond the capabilities of physical vehicles. $[8,9]$ published an equation in 2000 in attempt to resolve the shortcomings of Gipp's model by utilizing multiple sensitivity parameters and other methods; the model contains an acceleration strategy with a braking strategy to cover high and sparse traffic flow density.

[10] described an overview of the microscopic approach to modeling traffic and then elaborate on the various concepts associated with it. [11] introduced the assumptions about real driving behavior such as keeping a safe distance from the leading vehicle, driving at the desired speed, or preferring accelerations to be within a comfortable range. [12] researched the microscopic modeling of human and automated driving: towards traffic-adaptive cruise control, emphasizing on how operations can be improved by way of intelligent transportation systems (ITS). The work is based on microscopic traffic flow theory and the findings demonstrated the impact of the individual driver behavior on the overall traffic dynamics. In [13], mobility and traffic density of vehicles were considered, the focus of the research was on studying the effect of the traffic flow density on the delay of emergency message dissemination. The paper submitted that the common way of emergency message dissemination in VANETs is broadcasting. To overcome the broadcasting storm problem and improve the scalability of such large networks, he adopted a node cluster based broadcasting mechanism and came up with a mathematical model for the broadcasting delay which accounts for the randomness in user mobility and matches the highly dynamic nature of VANETs. Investigation on the minimum cluster size that achieves acceptable message delivery latency shows that network control and performance parameters are dependent on the traffic density. [14] investigated the effect of the density of cars on the overall interactions of the vehicles along a given distance of the road in their mathematical model for the study of traffic flow on the highways. It was observed that the density of cars per mile affects the net rate of interaction between them.

\section{RESEARCH METHODOLOGY}

\section{The Modeling Concept}

The basic concept of our model is from classic Newtonian mechanics, where acceleration may be regarded as the response of a matter to the stimulus it receives in the form of the force from the interaction with other particles in the system. In relation to this, the assumption is that drivers react in a specific way to a stimulus from the vehicle(s) directly ahead (or, in some cases, behind) them; it is such that drivers' response is directly proportional to the stimulus received. (Response) ${ }_{n} \propto$ (Stimulus) ${ }_{n}$

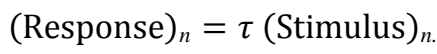

Where $\tau$ is the sensitivity factor, equation (1) can be succinctly expressed as we have in equation 2 .

$$
\text { Response }=\text { Sensitivity } \times \text { Stimulus. }
$$

Where Response is taken to be the acceleration of the following vehicle; for an $n^{\text {th }}$ vehicle say $n=1,2, \ldots$, each driver responds to the surrounding traffic conditions only by accelerating or decelerating the vehicle, which indicate correlation between the response of a driver and the relative speed of his vehicle and the one he is following; The Sensitivity factor most commonly, it is chosen as a constant or as inversely proportional to the spacing between consecutive vehicles but this is really a question of stability and steady-state flow; There are differences in views regarding the nature of the stimulus in traffic; the stimulus may be composed of the speed of the vehicle, relative position, distance headway and so on; hence, it is not a single variable, but a function and can be represented as in equation 3 ,

$$
a_{n}(t)=f_{s t i}\left(v_{n}, \Delta x_{n}, \Delta v_{n}\right)
$$

where $f_{s t i}$ is the stimulus function that depends on the speed of the current vehicle, relative position and speed with the front vehicle.

\section{The Modeling Notation}

From the points of view of safety, capacity and level of service, the Longitudinal spacing of vehicles are of particular importance. The longitudinal space occupied by a vehicle depends on the physical dimensions of the vehicles as well as the gaps between vehicles. For measuring this longitudinal space, two microscopic measures are used, Distance Headway and Distance Gap. Distance Headway is defined as the distance from a selected point (usually front bumper) on the leading vehicle to the corresponding point on the following vehicles. This includes the length of the leading vehicle 
and the gap length between the leading and the following vehicles.

The various notations used in this work and has often been used in Microscopic modeling (also known as carfollowing modeling) of traffic flow needs to be explained. The leading vehicle is denoted as $n$ and the following vehicle as $n+1$. Two characteristics at time instant $t$ are of importance; location and speed. Location and speed of the leading vehicle at time instant $t$ are represented by $x_{n}(t)$ and $v_{n}(t)$ respectively. Similarly, the location and speed of the following vehicle are denoted by $x_{n+1}(t)$ and $v_{n+1}(t)$ respectively. The following vehicle is assumed to accelerate at time $t+$ $\Delta \mathrm{T}$ and not at $t$, where $\Delta \mathrm{T}$ is the interval of time required for a driver to react to a changing situation. The gap between the leading and the following vehicle is therefore $x_{n}(t)-x_{n+1}(t)$. The notation for this modeling is shown in figure 1 .
Now, let $\Delta x_{n+1}(t)$ be the gap available for $(n+1)^{\text {th }}$ vehicle, and let $\Delta x_{\text {safe }}$ be the safe distance, $v_{n+1}(t)$ and $v_{n}(t)$ be the velocities, then it can be deduced that the gap required is given by,

$$
\Delta x_{n+1}(t)=\Delta x_{\text {safe }}+\tau v_{n+1}(t) .
$$

where $\tau$ is a sensitivity coefficient. Recall from Figure 1 ,

$$
\Delta x_{n+1}(t)=x_{n}(t)-x_{n+1}(t) .
$$

Thus equation (4) can be re-written as

$$
x_{n}(t)-x_{n+1}(t)=\Delta x_{\text {safe }}+\tau v_{n+1}(t)
$$

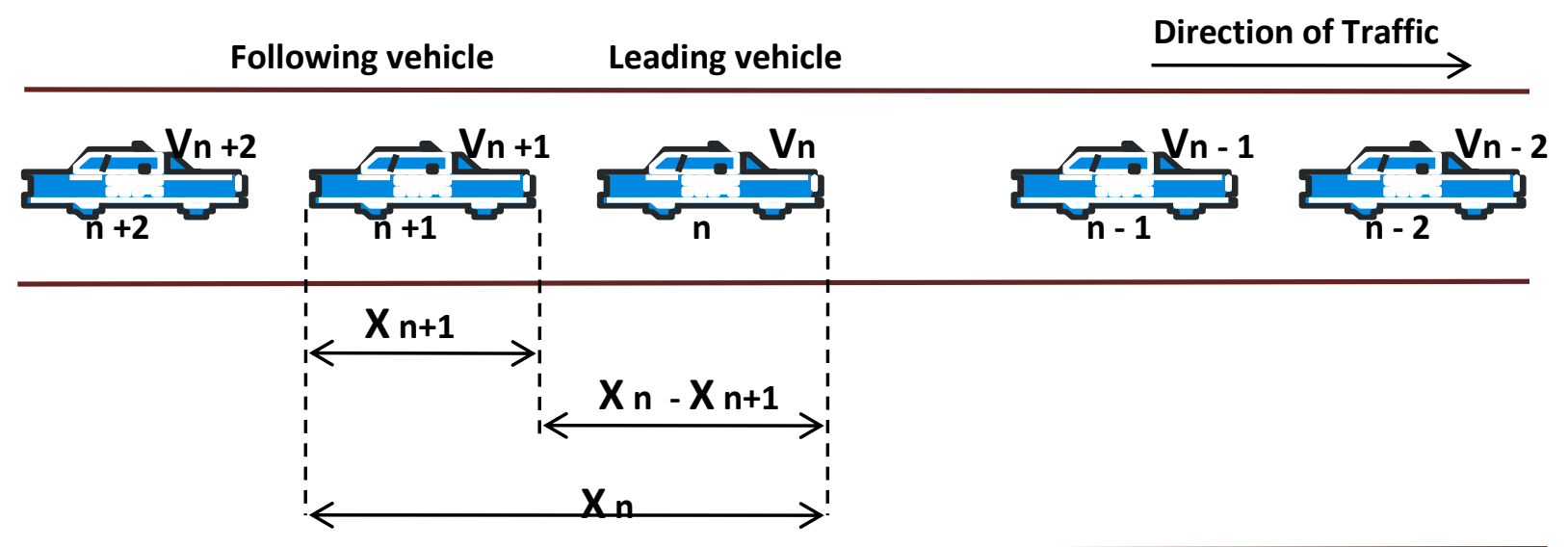

Figure 1: Notation for the Modeling

\section{Modeling the Traffic Flow}

The common everyday traffic scenarios were taken into consideration. A good instance which also forms the bases for this modeling is the following state which is encountered in everyday traffic scenario, with medium to high vehicle density. In such state, a vehicle's velocity and acceleration is largely determined by the vehicle in front of it whereby a driver attempts to maintain a minimum and maximum vehicle gap (or time gap) between themselves and the lead vehicle and the driver while approaching a stopped or attempting to stop using various degrees of braking force in an attempt to avoid colliding with the object in front of it.

For this modeling, the following four assumptions were made:

a) The higher the speed of the vehicle, the higher will be the spacing between the vehicles.

b) To avoid a collision, the driver must maintain a safe distance from the vehicle ahead.

c) A driver will react to the change in speed of the front vehicle after a time gap called the reaction time during which the follower perceives the change in speed and react to it.

d) Vehicle position and speed are governed by Newton's laws of motion.
Differentiating equation (6) with respect to time, result to

$$
\begin{aligned}
& \frac{d}{d t}\left(x_{n}(t)-x_{n+1}(t)\right)=\frac{d}{d t}\left(\Delta x_{\text {safe }}+\tau v_{n+1}(t)\right), \\
& v_{n}(t)-v_{n+1}(t)=\tau . a_{n+1}(t) \\
& a_{n+1}(t)=\frac{1}{\tau}\left[v_{n}(t)-v_{n+1}(t)\right] .
\end{aligned}
$$

To determine the sensitivity factor $\tau$, as earlier mentioned, it can be said to be inversely proportional to the spacing between consecutive vehicles but this is really a question of stability and steady-state flow. Expressing this in a mathematical terms with the view of Figure (1) in mind,

$$
x_{n+1}(t+\Delta T)=\tau\left(x_{n}(t)-x_{n+1}(t)\right) .
$$

where $x_{n+1}$ is the position of the following vehicle (as in Figure 1), $\Delta T$ is the time lag, which

is the reaction time of the driver, and $\tau$ is the sensitivity factor.

Consequently, it is also worth mentioning that equation (8) can be generalized to hold for more vehicles; it can be extended to let a vehicle respond to more vehicles than just the one right in front of it. If one wants to, for instance, include also the vehicle right in front of the currently leading vehicle, this results into: 


$$
\begin{aligned}
& x_{n+1}(t+\Delta T)=\tau_{1}\left(x_{n}(t)-x_{n+1}(t)\right)+\tau_{2} \\
& \left(x_{n-1}(t)-x_{n+1}(t)\right) .
\end{aligned}
$$

The sum of terms of equation (9) can be expressed as:

$x_{n+1}(t+\Delta T)=\tau_{1}\left(x_{n}(t)-x_{n+1}(t)\right)+\tau_{2}\left(x_{n-1}(t)-\right.$

$\left.x_{n+1}(t)\right)+\tau_{3}\left(x_{n-2}(t)-x_{n+1}(t)\right)+\ldots+\tau_{n}$

$\left(x_{n-n}(t)-x_{n+n}(t)\right) .=\sum_{n=1}^{k}\left[\tau_{k}\left(x_{k-n}(t)-x_{k+n}(t)\right)\right]$. (10)

If traffic moves at a constant speed, then equation (8) is always satisfied; obviously, there is no unique steadystate equation. Transitions from one steady state to another, after a change of velocity of the leading vehicle, can be achieved by integrating equation (8) on both sides. Doing this disposes of $\Delta T$ and generates a relationship between the spacing and speed of vehicles in a steady-state stream.

From the illustration in figure 1, this definition can, therefore, be made:

$$
x_{n}(t)-x_{n+1}(t) \stackrel{\text { def }}{=} l_{n}(t)
$$

Let $l_{n}(t)$ be the relative spacing between the leading and the following vehicle.

Integrating equation (8) with respect to $\tau$ equal to a constant, it now follows that

$\int_{-\infty}^{\infty}\left(x_{n+1}(t+\Delta T)\right) d t=\tau \int_{-\infty}^{\infty}\left(x_{n}(t)-x_{n+1}(t)\right) d t$, (11)

$$
\begin{aligned}
& {\left[x_{n+1}(t+\Delta T)\right]_{-\infty}^{\infty}=\tau\left[x_{n}(t)-x_{n+1}(t)\right]^{\infty}{ }_{-\infty},} \\
& v_{n+1}(t+\Delta T)=\tau\left(x_{n}(t)-x_{n+1}(t)\right) . \\
& \quad v_{\text {finish }}-v_{\text {Start }}=\tau\left(l_{n \text { finish }}-l_{n \text { start }}\right) .
\end{aligned}
$$

Clearly, the $\left(l_{n \text { finish }}-l_{n \text { start }}\right)$ part of the right hand side of equation (13) has the unit of length thus, inverting $l_{n}(t)$ gives $\frac{1}{\text { length }}$ which happens to have the same unit as that for density, $\rho$ hence, equation (13) can be re-written as

$$
v_{\text {finish }}-v_{\text {start }}=\tau\left[\frac{1}{\rho_{\text {nfinish }}}-\frac{1}{\rho_{n \text { start }}}\right] .
$$

Consequently, assuming the road is jam-packed when the analysis began, then $\rho_{n \text { start }}=\rho_{\max }$ and $v_{\text {start }}=0$. Generalising and setting $v=v_{\text {finish }}$, and $\rho=\rho_{\text {finish }}$ equation (14) becomes

$$
v=\tau\left[\frac{1}{\rho}-\frac{1}{\rho_{\max }}\right] \text {. }
$$

Therefore, the sensitivity coefficient $\tau$ can either be derived from equation (12), (13), (14) and (15) depending on the properties needed to be considered. From equation (12), the sensitivity coefficient $\tau$ can be derived as,

$$
\tau=\left[\frac{v_{n+1}(t+\Delta T)}{\left(x_{n}(t)-x_{n+1}(t)\right)}\right] .
$$

Again, $\tau$ can also be derived from equation (14) in relation to vehicular speed and road density as,

$$
\tau=\left[\frac{v_{\text {finish }}-v_{\text {start }}}{\frac{1}{\rho_{\text {nfinish }}}-\frac{1}{\rho_{\text {nstart }}}}\right]
$$

And from equation (15), $\tau$ can as well be derived as

$$
\tau=\left[\frac{v}{\frac{1}{\rho}-\frac{1}{\rho_{\max }}}\right]
$$

\section{The Vehicular Ad-Hoc Network Scenario of Our} Model

It has been established by classical Newton mechanics that there is no important distinction between a static object and uniform motion object in a straight line; they may be regarded as the same state of motion seen by different observers, one moving at the same velocity as the object, the other moving at constant velocity with respect to the object. Thus, vehicles in VANET can be described as mobility in one spatial dimension. Figure 2 shows vehicle-to-vehicle and vehicle-to-roadside infrastructure communication scenario.

It can be recalled according to [15] that, in any VANET environment, calculating the transmission range and then finding the probability of packet delivery ratio (PDR) is important, thus, the transmission range for a Free Space wireless channel model can be calculated using the Friis Transmission equation, the propagation model is to predict received signal strength when the transmitter and receiver have a clear, unobstructed line of sight radio link. Equation (19) describes the Friis Transmission equation given as:

$$
P_{r}=\frac{P_{t} G_{t} G_{r} \lambda^{2}}{(4 \pi)^{2} d^{2} L}
$$

where $P_{r}$ and $P_{t}$ are receive and transmit powers respectively, $G_{t}$ and $G_{r}$ are gains of transmit and receive antennas respectively, $\lambda$ is the wavelength, $L$ is the system loss factor not related to propagation and $d$ is the distance between transmitting and receiving nodes. Considering proper propagation scheme, wireless channel model and combining schemes at the receiver will improve the Quality of Service (QoS) in VANET. Therefore, it can be deduced that, if the transmission power and antenna parameters of the OBU-RSU is properly designed, then the computational and processing time for secure message in VANET is largely a function of the algorithm of the cryptographic method, which must be significantly less than the established situation response time for the follow-thelead vehicle of this mathematical model. 

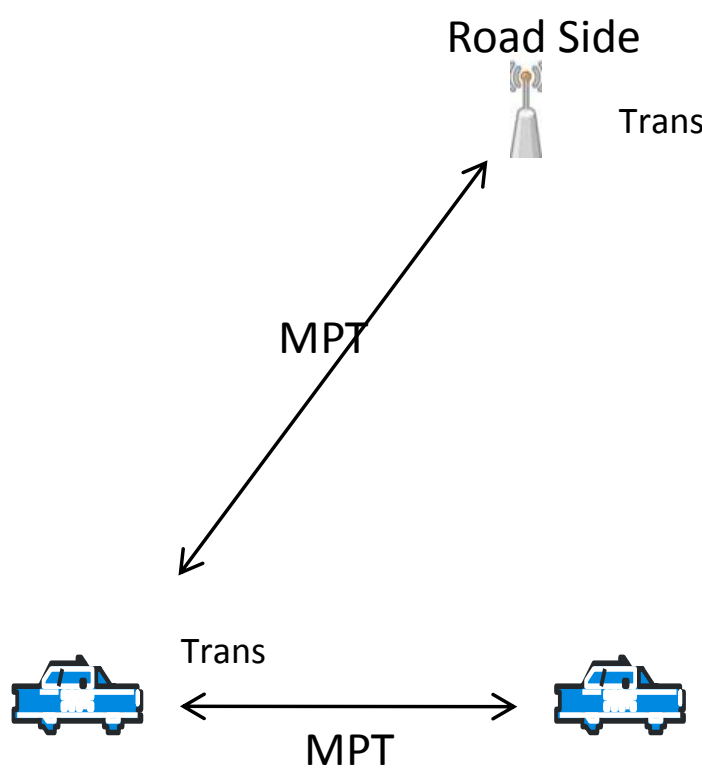

Trans

MPT = Message Processing Time

SRT = Situation Response Time

Figure 2: Vehicle-to-Vehicle and Vehicle-to-Road Side Infrastructure Communication

\section{The Model Equation Formulation}

The governing equations of the traffic flow can be developed as below. Let $\Delta T$ is the reaction time, and $\Delta t$ is the updating time, the governing equations can be written as,

$$
\begin{gathered}
v_{n}(t)=v_{n}(t-\Delta t)+a_{n}(t-\Delta t) \times \Delta t \\
x_{n}(t)=x_{n}(t-\Delta t)+v_{n}(t-\Delta t) \times \Delta t+\frac{1}{2} a_{n}(t-
\end{gathered}
$$
$\Delta t) \Delta t^{2}$

$$
\begin{aligned}
& a_{n+1}(t-\Delta t)=\left[\frac{v_{n+1}(t+\Delta T)}{\left[x_{n}(t)-x_{n+1}(t)\right]}\right]\left[v_{n}(t-\Delta t)-\right. \\
& \left.v_{n+1}(t-\Delta t)\right] .
\end{aligned}
$$

The equation (20) is a simulation version of Newton's law of motion $v=u+a t$, equation (21) is the simulation version of another Newton's law of motion equation $s=u t+\frac{1}{2} a t^{2}$ and equation (22) is derived from equation (7) of this modeling. The acceleration of the following vehicle depends upon the relative velocity of the leading and the following vehicle, sensitivity coefficient and the gap between the vehicles.

\section{RESULT AND DISCUSSION}

\subsection{Application of Problem to the Model}

Let say, a leading vehicle is moving with zero acceleration for two seconds from time $t=0$. Then he accelerates by $1 \mathrm{~m} / \mathrm{s}^{2}$ for 2 seconds and then decelerates by $1 \mathrm{~m} / \mathrm{s}^{2}$ for another 2 seconds. The initial speed is 20 $\mathrm{m} / \mathrm{s}$ and the initial location is $32 \mathrm{~m}$ from the reference point, and a vehicle is following this vehicle with initial speed $20 \mathrm{~m} / \mathrm{s}$, with a position at zero. The simulation of the behavior of the following vehicle using our mathematical model determines the reaction time of the following vehicle at a scan interval of 0.5 seconds for a period of 5 seconds. The solution of the model is described in section 4.1.1.

\subsubsection{Solution of the Model}

From the application problem, it can be deduced that at time $t=0$, the leading vehicle has a velocity of $20 \mathrm{~m} / \mathrm{s}$ and located at a distance of 32 meters from the reference point. The following vehicle is also having the same velocity of $20 \mathrm{~m} / \mathrm{s}$ and located at the same reference point. Since the velocity is the same for both, $d v=0$. At time $t=0$, the leading vehicle is said to be having zero acceleration, and hence it has a constant speed. The location of the leading vehicle can be found out from equation (21) as, $x_{n}(t)=x_{n}(t-\Delta t)+v_{n}(t-\Delta t) \times$ $\Delta t+\frac{1}{2} a_{n}(t-\Delta t) \Delta t^{2} \quad$ Where $x_{n}(t-$ $\Delta t)=32 ; v_{n}(t-\Delta t)=20 ; \Delta t=0.5$ and $\frac{1}{2} a_{n}(t-\Delta t)$ $\Delta t^{2}=0$ because acceleration, $\mathrm{a}=0$. Therefore, $x_{n}(t)=$ $32+20 \times 0.5=42$ metres. Similarly, the following vehicle is not accelerating and is maintaining the same speed. The location of the following vehicle is also derived from equation (21) as, $x_{n}(t)=x_{n}(t-\Delta t)+$ $v_{n}(t-\Delta t) \times \Delta t+\frac{1}{2} a_{n}(t-\Delta t) \Delta t^{2}$. Where $x_{n}(t-\Delta t)$ $=0 ; v_{n}(t-\Delta t)=20 ; \Delta t=0.5$ and $\frac{1}{2} a_{n}(t-\Delta t) \Delta t^{2}=0$ because acceleration, $\mathrm{a}=0$. Therefore, $x_{n}(t)=0+20 \times$ $0.5=10$ metres. Therefore, $d x=42-10=32$ metres.

These steps are repeated until $t=1.5$ seconds. At time $t$ $=2$ seconds, leading vehicle accelerates at the rate of 1 $\mathrm{m} / \mathrm{s}^{2}$ and continues to accelerate for 2 seconds. After that, it decelerates for a period of two seconds. At $t=$ 2.5 seconds, the velocity of leading vehicle based on equation (20), $v_{n}(t)=v_{n}(t-\Delta t)+a_{n}(t-\Delta t) \times \Delta t$. Where $v_{n}(t-\Delta t)=20, a_{n}(t-\Delta t)=1$, and $\Delta t=0.5$, the velocity changes to $20.5 \mathrm{~m} / \mathrm{s}$. Thus $d v$ becomes 0.5 $\mathrm{m} / \mathrm{s}$ at 2.5 seconds, $d x$ also changes since the position of leading vehicle changes. The following vehicle responds to the leading vehicle's change in acceleration at $t=5$ seconds based on equation (22), $a_{n+1}(t-\Delta t)=$ $\left[\frac{v_{n+1}(t+\Delta T)}{\left[x_{n}(t)-x_{n+1}(t)\right]}\right] \quad\left[v_{n}(t-\Delta t)-v_{n+1}(t-\Delta t)\right]$ which translate to the ratio of the difference in velocities between the leading and following vehicle denoted as $d v$ and the difference in displacement between the leading and following vehicle denoted as $d x ; a=\frac{d v}{d x}=$ $\frac{1}{35}=0.03 \mathrm{~m} / \mathrm{s}^{2}$. It therefore shows that the current acceleration of the following vehicle depends on $d v$ and reaction time in split seconds.

The following vehicle will change the speed at the next time interval. i.e., at time $t=4$ seconds. The speed of the following vehicle at $t=4$ seconds is given by equation (20), $v_{n}(t)=v_{n}(t-\Delta t)+a_{n}(t-\Delta t) \times \Delta t$. Where $v_{n}(t-\Delta t)=20, a_{n}(t-\Delta t)=0.03$ (which is the situation response acceleration of the leading vehicle recorded at time $t=5$ ), and $\Delta t=0.5$, as $v_{n}(t)=20+$ 
$0.03 \times 0.5=20.02 \mathrm{~m} / \mathrm{s}$. The location of the following vehicle at $t=4$ seconds is given by equation (21), $x_{n}(t)=x_{n}(t-\Delta t)+v_{n}(t-\Delta t) \times \Delta t+\frac{1}{2} a_{n}(t-\Delta t)$ $\Delta t^{2}$. Where $x_{n}(t-\Delta t)=0 ; v_{n}(t-\Delta t)=20 ; \Delta t=0.5$ and $\frac{1}{2} a_{n}(t-\Delta t) \Delta t^{2}=0$, as $x=70+20 \times 0.5+\frac{1}{2} \times$ $0.03 \times 0.5^{2}=80.004$ metres. These steps are followed for all the cells of the table.

\subsubsection{Result Analysis}

Table 1 shows the result of the application of the developed model in tabular form. Figure 3 shows the relationship between the speed of the Leading Vehicle and the Following Vehicle with reference to Time, the graph shows a level of safety between the two drivers on the road. Figure 4 shows the relationship between the position of the Leading Vehicle and the Following Vehicle with respect to Time.

Table 1: Result of Application of Problem to the Model

\begin{tabular}{lllllll}
\hline $\begin{array}{l}\text { Time } \\
(\text { secs })\end{array}$ & \multicolumn{2}{l}{ Leading Vehicle } & \multicolumn{4}{c}{ Following Vehicle } \\
$T$ & $a(t)$ & $v(t)$ & $x(t)$ & $a(t)$ & $v(t)$ & $x(t)$ \\
\hline 0 & 0 & 20 & 32 & 0 & 20 & 0 \\
0.5 & 0 & 20 & 42 & 0 & 20 & 10 \\
1 & 0 & 20 & 52 & 0 & 20 & 20 \\
1.5 & 0 & 20 & 62 & 0 & 20 & 30 \\
2 & 1 & 20 & 72 & 0 & 20 & 40 \\
2.5 & 1 & 20.50 & 825 & 0 & 20 & 50 \\
3 & 1 & 21 & 95 & 0 & 20 & 60 \\
5 & 1 & 21.5 & 1075 & 0.03 & 20 & 70 \\
4 & -1 & 22 & 114 & 0.04 & 20.02 & 80.0 \\
4.5 & -1 & 21.5 & 124.25 & 0.06 & 20.04 & 90.0 \\
5 & -1 & 21 & 134.5 & 0.04 & 20.06 & 100.0 \\
\hline
\end{tabular}

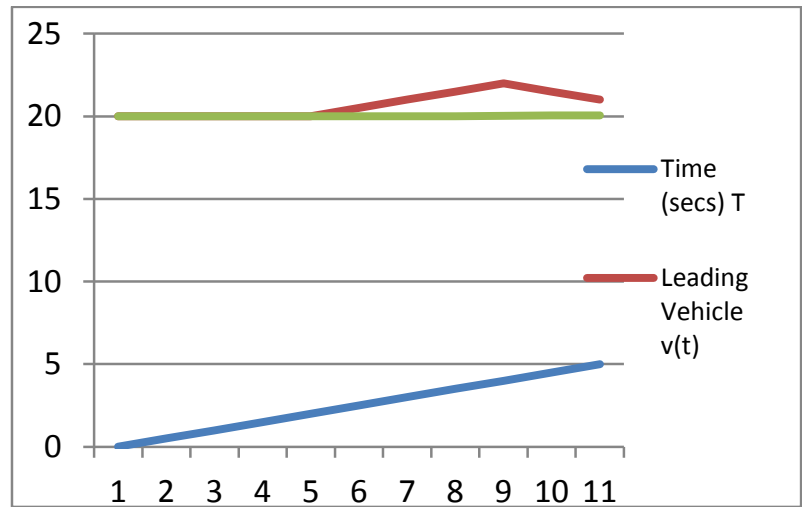

Figure 3: The Relationship between the Speed of the Leading Vehicle and Following Vehicle versus Time

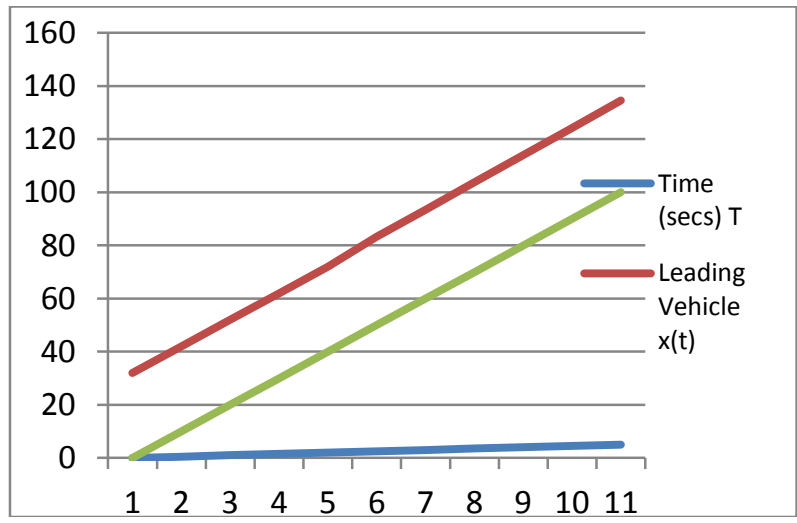

Figure 4: The Relationship between the Position of the Leading Vehicle and the Following Vehicle versus Time

\section{CONCLUSION}

While there is no unified theory to describe traffic flow; the difficulty lies in modeling any type of phenomena when human elements are present. Humans change their driving style constantly, which may prevent anyone model from ever describing complex traffic patterns with complete accuracy; this work has successfully models each vehicle in a chain of others and how it responds to the traffic around it using Microscopic (also known as car-following) method for modeling traffic flow; driver- to-driver and driver-to-road interactions within traffic stream and the interaction between a driver and another driver on road were considered. This model determines the minimum response time required for a vehicle in VANET to respond and communicate situations on the road.

A simulated scenario was carried out for two vehicles, a leading vehicle and following vehicle. The result shows that with an average of 32 meters apart with an average difference in velocity of $1.23 \mathrm{~m} / \mathrm{s}$, a minimum of 0.9 secs is required for efficient situation response communication to ensue between them. It was therefore deduced that, if the transmission power and antenna parameters of the OBU-RSU is properly designed, then the computational and processing time for secure message in VANET is largely a function of the algorithm of the cryptographic method, which must be significantly less than the established situation response time for the follow-the-lead vehicle of this mathematical model.

\section{REFERENCES}

[1] Report on Accidents in Africa" WHO. 2013

[2] G. Chen, "Road Traffic Safety in Africa Countries Status, Trend, Contributing Factor, Counter Measures And Challenges". International Journal of Injury Control and Safety Promotion, vol. 17, no. 4, pp. 247 - 255. 2010

[3] O. Agbonkhese, G. L. Yisa, E. G. Agbonkhese, D. O. Akanbi, E. O. Aka, and E. B. Mondigha, "Road Traffic Accidents in Nigeria: Causes and Preventive Measures". Civil and Environmental Research, vol. 3, no. 13, 2013

[4] V. N. Ukoji, "Trends and Pattern Fatal of Road Accidents in Nigeria". French Institute for Research in Africa (IFRA Nigeria) Working Paper Series, no. 35. 2014

[5] G. Samara, W. A. H. Al-Salihy, R. Sures, "Security Analysis of Vehicular Ad Hoc Networks (VANET)". Second International Conference on Network Applications, Protocols and Services, pp. 2-32. 2010

[6] M. Khatri, and S. Malhotra, "An Insight Overview Of Issues And Challenges In Vehicular Adhoc Network," Journal of Global Research In Computer Science, vol. 2, no. 12, pp. 8-1. 2011

[7] A. Kesting, and M. Treiber, "Calibrating Car-Following Models by Using Trajectory Data: Methodological Study. Transportation Research Record": Journal of the Transportation Research Board, vol. 2088 no.1, pp. 148-56. 2008

[8] M. Treiber, H. Ansgar, and H. Dirk, "Congested Traffic States in Empirical Observations and Microscopic Simulations". Physical Review vol. 62, no.2, pp. 1805-824. 2000,

[9] M. Treiber, "Microsimulation of Road Traffic Flow. Technische Universitat Dresden. Web." http://vwisb7.vkw.tudresden.de/ treiber/MicroApplet/index.html. 2011

[10] T. V. Mathew, and K. V. K. Rao, "Introduction to Transportation Engineering: Microscopic Traffic Flow Modeling," NPTEL. 2007

[11] A. Kesting, and M. Treiber, "Calibrating Car-Following Models by Using Trajectory Data: Methodological Study". 
Transportation Research Record: Journal of the Transportation Research Board, vol. 2088 no.1, pp. 148-56. 2008

[12] A. Kesting, "Microscopic Modeling of Human and Automated Driving: Towards Traffic Adaptive Cruise Control". Doctoral Thesis, Faculty of Traffic Sciences, Technische Universitat Dresden, Germany. 2008

[13] K. H. Abboud, "Modeling and Analysis of Emergency Messaging Delay in Vehicular Ad hoc Networks." Master of Applied Science in Electrical and Computer Engineering Thesis, University of Waterloo, Ontario, Canada. 2009
[14] S. E. Fadugba, J. T. Okunola A. O. Ajayi, and O. H. Edogbanya, "On Mathematical Model For The Study Of Traffic Flow On The High Ways". International Journal of Mathematical Research, Vol. 3, No. 3, pp. 25-36. 2014

[15] D. M. Pozar, "Microwave Engineering", 3rd Edition. New York: Wiley, 